\title{
Radiating Elements for Shared Aperture Tx/Rx Phased Arrays at K/Ka Band
}

\author{
A.I Sandhu, E. Arnieri, Member, IEEE, G. Amendola, Senior Member, IEEE, \\ L. Boccia, Member, IEEE, E. Meniconi and V. Ziegler Senior Member, IEEE
}

\begin{abstract}
A dual band, Tx/Rx, self-diplexing phased array is presented. The antenna has been designed to cover $\mathrm{Tx} / \mathrm{Rx}$ satellite communications at $\mathrm{K} / \mathrm{Ka}$ band with a frequency ratio $1.5: 1$. To obtain dual band operations with a single radiating surface, a novel dual band radiator is adopted and placed in a configuration in which dual band and single band elements are interleaved. The proposed configuration reduces the number of radiating elements required by other solutions while avoiding the insurgence of grating lobes. The tightly packed arrangement of the elements poses many integration issues, which are solved with a novel integration technique. The array elements are optimized to scan the beam in excess of $50^{\circ}$ in both bands. A subarray with $49 \mathrm{Rx}$ elements and 105 Tx elements was built and measured confirming the results obtained in simulations.
\end{abstract}

Index Terms-Microstrip antennas, multi-frequency antennas, phased arrays, planar arrays

\section{INTRODUCTION}

$\mathrm{M}$ ODERN communication systems require highly integrated user terminals operating in the millimeter wave region embedding antennas with beam steering capability. Examples include $\mathrm{K} / \mathrm{Ka}$ band SatCom on-the-move (SOTM) terminals [1] and $60 \mathrm{GHz}$ high-capacity communications in complex indoor scenarios [2], [3]. It is also foreseen that 5G technology for next generation wireless communication networks will rely on sophisticated beam-forming capabilities in which hand held terminals and base-stations communicate through optimum line-of-sight/ Non-line-of-sight (LOS/NLOS) millimeter wave links [4]. In all these applications, radiation boards, interconnects, beam forming networks, Tx and Rx modules and other circuitry have to be included in a reduced volume increasing the complexity of the integration process. A significant milestone in this direction has been achieved with the recent introduction of multifunctional chipsets capable to contain many reconfigurable transmit $(\mathrm{Tx})$ and Receive $(\mathrm{Rx})$ chains. However, there are still functionalities that are intrinsically space consuming. At the antenna level one of the main issues is that $\mathrm{Rx}$ and Tx operations are usually obtained with two separate radiating apertures thus considerably increasing the actual space occupied by the system. The design of arrays able to integrate both Tx and Rx operation in a single aperture is cumbersome, albeit essential. Two solutions may be devised: arrays of elements with a bandwidth large enough to cover both Tx and Rx or arrays of dual band elements. The first

Manuscript received November 15, 2015. This work was supported by EU commission under the FP7 project FLEXWIN. E. Arnieri, G. Amendola and L. Boccia are with the University of Calabria, Rende (Italy), (e-mail: solution has been largely explored [5], [6]. For example, arrays of tightly coupled dipoles with ultra-wide band performance have been recently studied [7] for Ku-band SOTM applications showing excellent scanning behavior in a wide angular range. Nevertheless, this approach suffers from three inherent drawbacks. Firstly, the tightly coupled dipoles configuration corresponds to a very small cell size (less than 0.3 wavelength at the highest frequency) which makes the integration of the TR modules difficult. Secondly, the reduced cell size results in an increased number of elements and, consequently, of control signals thus making significantly more critical the manifold design. Thirdly, as the array is not self-duplexing some mechanisms to discriminate between the two bands would be required.

Other dual band phased-arrays have been proposed in literature mainly for Synthetic Aperture Radar instruments operating at multiple bands [8], [9]. Small multiband arrays have been also designed for mobile telecommunications base-stations [10]. Wide-angle scanning was recently taken into account in [11], [12] designing dual-band radiating elements for $\mathrm{X} / \mathrm{Ku}$ phased arrays with beam steering capability down to 60/50 degrees.

In this paper, a new concept of compact dual-band phased array supporting wide-angle beam steering is presented. The

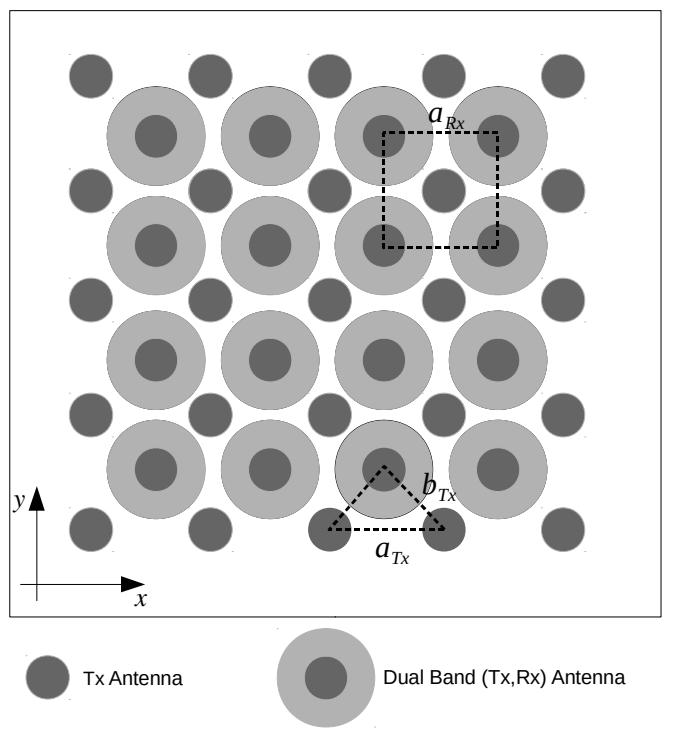

Fig. 1. Dual-band array lattice with interleaved Tx and Rx elements.

luigi.boccia@unical.it). A. I. Sandhu is now with KAUST, Jeddah (Saudi Arabia). E. Meniconi is now with Qorvo, Inc., Munich (Germany) and V. Ziegler is with Airbus Group Innovations, Munich (Germany). 


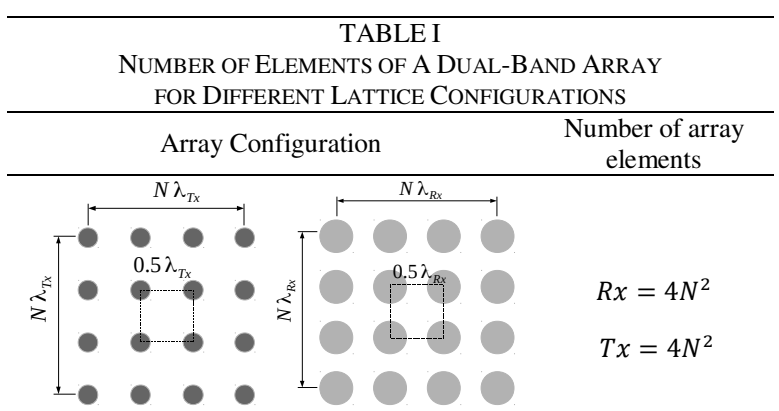

Two single-band arrays with square cells

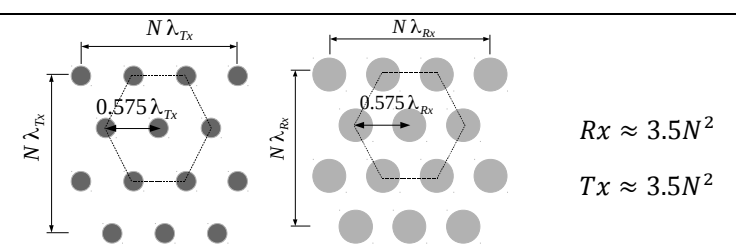

Two single-band arrays with hexagonal cells

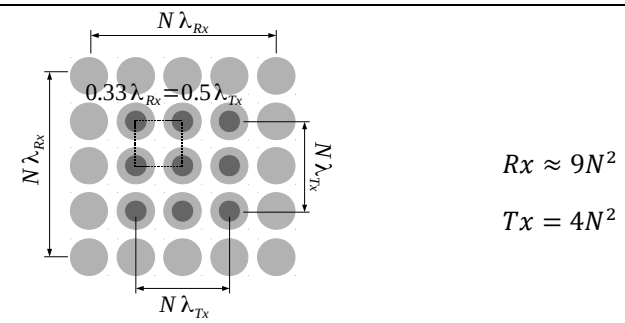

Shared aperture array with square unit cells.

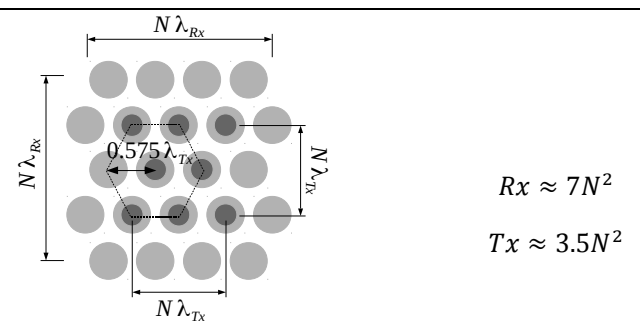

Shared aperture array with hexagonal cells $0.575 \lambda$ at $30 \mathrm{GHz}$

\begin{tabular}{lcc}
\hline Shared aperture array & (interleaved & $R x$ \\
configuration in Fig. 1) & & $T x \approx 4 N^{2}$ \\
& &
\end{tabular}

The number of array elements (in the Tx and Rx band) is calculated for an aperture equal to $(\mathrm{N} \lambda)^{2}$ where $\mathrm{N}$ is an integer number and $\lambda$ is the free-space wavelength on each band.

proposed configuration mainly targets applications with large frequency ratios, wide scanning range and full electronic control. The array illustrated in this work has been conceived taking as a reference case $\mathrm{K} / \mathrm{Ka}$-band SOTM applications (Rx 20.2-21.2 GHz, Tx: $29.5-30.8 \mathrm{GHz})$ with a scan angle of $\pm 60^{\circ}$. The proposed array has been designed to be integrated with multicore, $\mathrm{SiGe}$ BiCMOS $3.8 \times 3.8 \mathrm{~mm}^{\wedge} 2$ chips containing two $\mathrm{Rx}$ and four Tx chains designed in the framework of the EU FP7 project FLEXWIN [13], [14].

For the application at hand, due to the tight arrangement of the radiating elements and to the ratio between the $\mathrm{Tx}$ and $\mathrm{Rx}$ center frequencies (1.5:1), the design of the radiating elements is particularly challenging for the following inherent reasons. Firstly, the array lattice and the cell size have to be selected to avoid grating lobes in the whole scanning region. Secondly, although the frequency ratio between the Tx and $\mathrm{Rx}$ bands could be covered with wide-band radiators they would not be self-duplexing thus making the chip integration significantly more complex [15]. On the other hand, the frequency ratio is not wide enough to facilitate the design of dual band elements. To solve the two problems, a novel radiating element based on coaxial printed rings is introduced in this paper. As it will be shown, the new element can be made compact enough to be embedded in arrays with small cell sizes, it can be optimized to achieve wide scan angle and it can be easily integrated with multi-core MMICs. Although the specific context of this work is related to $\mathrm{K} / \mathrm{Ka}$-band SOTM user terminals, its scope can be extended to other dual-band phased arrays with large frequency ratio and wide scanning.

The paper is organized as follows. The concept of superimposed array and the lattice definitions are described in Section II. The dual- and single-band elements are presented in Section III. Simulated and measured results are shown in Section IV and V demonstrating very good performance in terms of scanning impedance in both the E- and H-plane.

\section{DuAl BAND ARRAy LatTice}

The definition of the array lattice influences the design of a dual-band phased array in several ways. The most direct impact is on the radiation performance. Indeed, the proper elements' location on the radiating surface is the main means to avoid grating lobes arising within the entire scanning range and in both bands. Moreover, from the array lattice depends also the number of elements present in a given radiating aperture and, in turn, the number of $\mathrm{Rx}$ and $\mathrm{Tx} \mathrm{RF}$ chains required to implement the electronic beam control. Finally, the inter-element spacing is also crucial to allow the integration of the active chips and to enable a feasible radiating element design.

Different strategies can be adopted to identify the best topology for the dual-band phased-array. For the case at hand, a comparative evaluation of different lattices was performed as

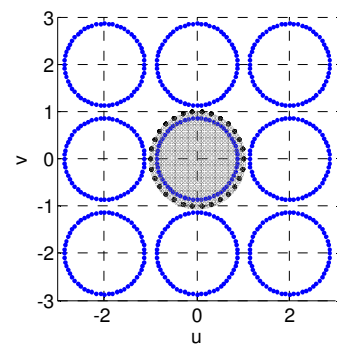

(a)

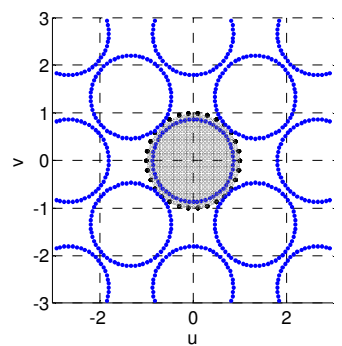

(b)
Fig. 2. Grating lobes diagrams for the $20 \mathrm{GHz}$ array on a rectangular lattice (a) and for the $30 \mathrm{GHz}$ array on a triangular lattice (b). Scan angle is $\theta_{s}=60^{\circ}$ in both cases. Shaded region included in black dots is the visible space. 
summarized in Table I. The radiating aperture was defined for each band in terms of the wavelength as $\left(N \lambda_{R x}\right)^{2}$ and $\left(N \lambda_{T x}\right)^{2}$ where $N$ is an integer number and where $\lambda_{R x}$ and $\lambda_{T x}$ are the Rx and Tx wavelengths respectively. The first example taken into account is a based on two single aperture uniform arrays, namely a square and a hexagonal lattice, designed to meet the grating lobes' constrain. A square array with a $0.5 \lambda \times$ $0.5 \lambda$ unit cell would require $4 N^{2}$ elements for each aperture whereas a hexagonal arrangement with a radius of $0.575 \lambda$ would limit the number of elements to about $3.5 N^{2}$. For a shared aperture $\mathrm{Tx} / \mathrm{Rx}$ phased array, the simplest configuration is to design dual-band elements small enough to be placed in a cell fulfilling the grating lobes' constrains at the highest frequency. As an example, dual-band radiating elements could be placed in a square lattice with a cell size of half a wavelength at $30 \mathrm{GHz}$ which will correspond to a cell of a third of a wavelength at $20 \mathrm{GHz}$, as it is shown in the third case presented in Table I. Although this solution fulfills the grating lobes' requirements, it would increase the number of required elements leaving limited space for the integration of the chips. On the other hand, a hexagonal lattice would reduce the number of required elements by $13 \%$ at both frequencies. As an alternative solution, the hybrid lattice shown in Fig. 1 is here proposed to limit the overall number of array elements and to keep the cell size at $20 \mathrm{GHz}$ and at $30 \mathrm{GHz}$ independent one from the other. The proposed solution is based on two arrays of $\mathrm{Rx}$ and of Tx elements superimposed. The Rx elements are placed on a square lattice with a cell size equal to $a_{R x}$ aligned to the $x-y$ axis. The Tx elements are placed on an isosceles triangular lattice having base $a_{T x}$ and height $b_{T x}$. The unit cell of the dual band lattice is constituted by the set of three radiators highlighted in the lower part of Fig. 1, namely one dual-band and two Tx antennas.

It is worth noticing that the proposed arrangement can be adapted to different dual-band arrays but it requires single band Tx interleaved with dual-band unit-cells. For the case at hand and for any other application having a frequency ratio of 1.5:1, the optimal configuration would lead to $a_{R x}=0.5 \lambda_{R x}=$ $0.75 \lambda_{T x}=a_{T x}$.

Both configurations fulfil the conditions on grating lobes derived as in [16]

$$
\begin{gathered}
a_{R x}<\frac{\lambda_{20}}{1+\sin \left(\theta_{S R x}\right)} \\
a_{T x}<\frac{\sqrt{2} \lambda_{30}}{1+\sin \left(\theta_{S T x}\right)}, \quad b_{T X}=a_{T X} / 2
\end{gathered}
$$

where $\theta_{S R x}$ and $\theta_{S T X}$ is the main beam elevation for the $\mathrm{Rx}$ and $\mathrm{Tx}$ array respectively. The second condition can be easily derived from the first one considering the triangular lattice as a square one rotated of $45^{\circ}$ and with a spacing of $0.53 \lambda_{T X}$. Considering a scan angle of $60^{\circ}$ at both bands (i.e. $\theta_{S R x}=$ $\theta_{S T X}=60^{\circ}$ ) it can be verified that grating lobes do not arise when $a_{R x}<0.539 \lambda_{R x}$ and $a_{T x}<0.7579 \lambda_{T x}$. As a further

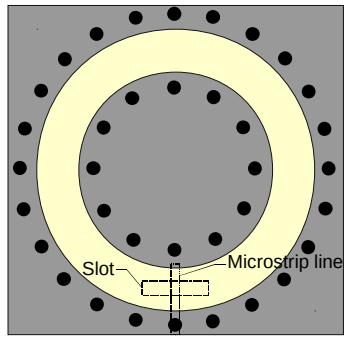

(a)

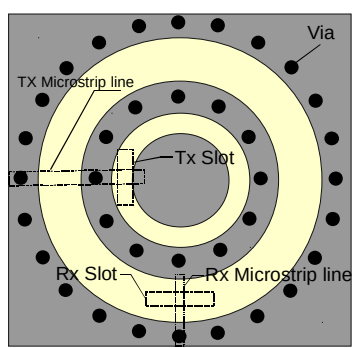

(c)

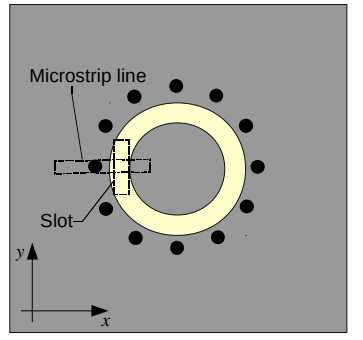

(b)

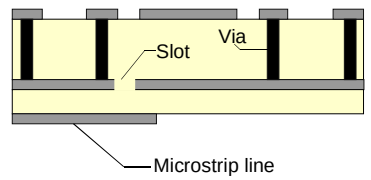

(d)
Fig. 3. Basic concept of the elementary cells required to implement the lattice of Fig. 1: a) single band Rx antenna; b) single band Tx antenna; c) dual Band Tx/Rx antenna; d) antenna stackup.

validation of the proposed configuration, grating lobe diagrams for both frequencies are shown in Fig. 2. As it can be observed, grating lobes are outside the visible region for scan angles $\theta_{s}<$ $60^{\circ}$ and for any azimuth angle.

\section{RADIATING ELEMENTS}

In this section are described the dual-band (Tx/Rx) and the single-band Tx radiating elements required to implement the previously introduced dual-band array lattice. In the first part, the basic concept of the array cells and their radiation characteristics is introduced while in the second part the design is actualized for the application at hand in an infinite-array context.

\section{A. Radiating element concept}

The design of the array elements required for the implementation of the lattice described in Fig. 1 is based on two single-band radiating elements as shown in Fig. 3. Both the Rx (Fig. 3-a) and the Tx (Fig. 3-b) single band radiators are made by an annular aperture surrounded by a cylindrical cage of metalized via holes forming a radiating cavity. These conducting walls help to minimize the impact of mutual coupling effects thus limiting the insurgence of side lobes. The antenna operating in the lower band ( $\mathrm{Rx}$ ) has an additional shorted inner boundary implemented through a coaxial ring of via holes. The inner pad of the Tx radiator is instead not grounded and it is used for fine frequency tuning and to better matching. Each ring apertures are electromagnetically coupled to a microstrip by means of a rectangular slot.

The two single band structures can be combined as shown in Fig. 3-c by accommodating the radiator of Fig. 3-b inside the one of Fig. 3-a in such a way that the inner wall of metallized vias is shared between the two elements. As already shown by 


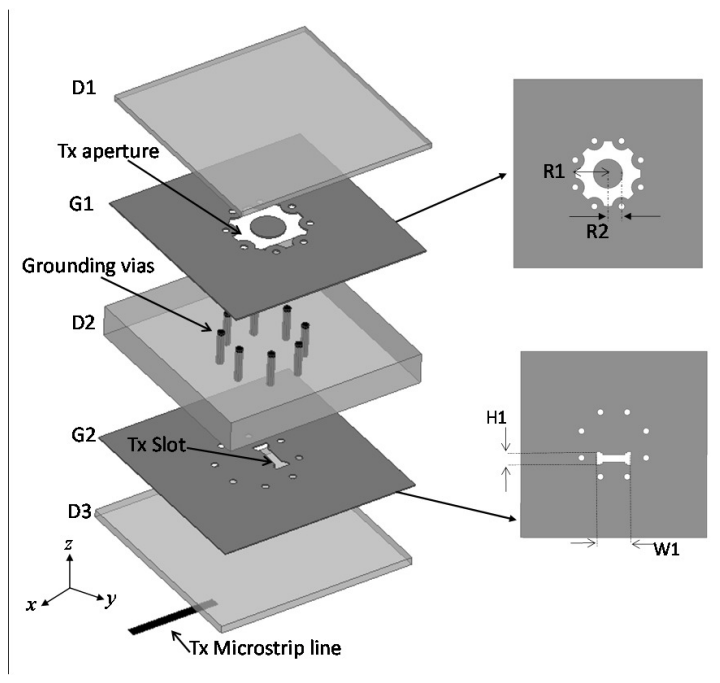

Fig. 4. 3D view of the of the Tx single-band element: $R 1=1.65 \mathrm{~mm}$, $\mathrm{R} 2=0.7 \mathrm{~mm}, \mathrm{~W} 1=1.4 \mathrm{~mm}, \mathrm{H} 1=1.05 \mathrm{~mm}$,

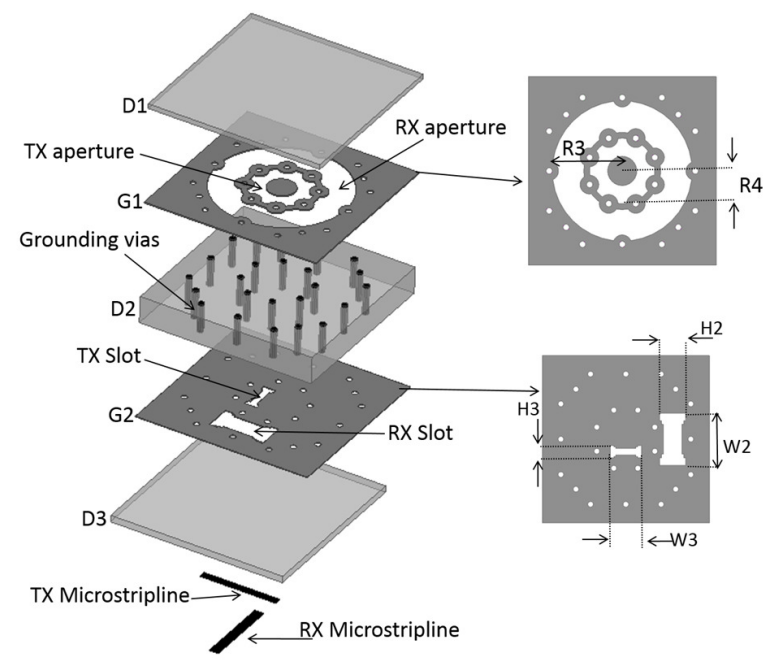

Fig. 5. 3D view of the dual band, Tx-Rx dual-band element: $\mathrm{R} 3=3.5 \mathrm{~mm}$, $\mathrm{R} 4=1.84 \mathrm{~mm}, \mathrm{~W} 2=2.8 \mathrm{~mm}, \mathrm{~W} 3=1.6 \mathrm{~mm}, \mathrm{H} 2=1.4 \mathrm{~mm}, \mathrm{H} 3=0.95 \mathrm{~mm}$.

the authors in [17], the dual-band antenna can be thus designed by optimizing the geometry so as the external and the internal antenna operate in the $\mathrm{Rx}$ and $\mathrm{Tx}$ band respectively. In the $\mathrm{Rx}$ case, due to the coaxial geometry, the antenna may propagate TEM, non-radiating, modes. However, if properly designed, a radiating TM mode can be excited [18].

The proposed configuration has several unique features which are explained as follows. Dual-band (Tx and $\mathrm{Rx}$ ) antennas and single-band Tx cells can be implemented as in Fig. 1 using the same 3-layer stack-up. The radiation properties of the Tx single-band radiator are similar to the ones in the dual-band configuration thus perfectly fitting the requirements of the array lattice proposed in Section II. Furthermore, the presence of the outer via cage is crucial in avoiding leakage and excitation of parallel plate mode

\begin{tabular}{|c|c|c|c|}
\hline Layer & Stack-up structure & Thickness & Material \\
\hline D1 & & $0,250 \mathrm{~mm}$ & FastriseDS \\
\hline G1 & & $0,033 \mathrm{~mm}$ & Copper \\
\hline D2 2 & V & $1,5 \mathrm{~mm}$ & Multiclad HF \\
\hline G2 & & $0,033 \mathrm{~mm}$ & Copper \\
\hline D3 & & $0,200 \mathrm{~mm}$ & Multiclad HF \\
\hline ML & Microstrip & $0,018 \mathrm{~mm}$ & Copper \\
\hline
\end{tabular}

Fig. 6. Cross sectional view of the single band and dual band element.

outside the radiator. Moreover, as it will be shown in the following paragraphs, the inner coaxial via cage allows the radiators in the two bands to be designed almost independently thus significantly simplifying the overall design process.

\section{B. Radiation characteristics}

The radiation properties of the antennas presented in the previous-subsection were evaluated by taking into account a simplified model of PCB multilayer circuit as shown in Fig. 4-6. Two types of radiating cells were taken into account, namely the single band (Tx) and the dual-band (Tx/Rx) cell. Antennas were fed through a dog-bone slot cut in the ground

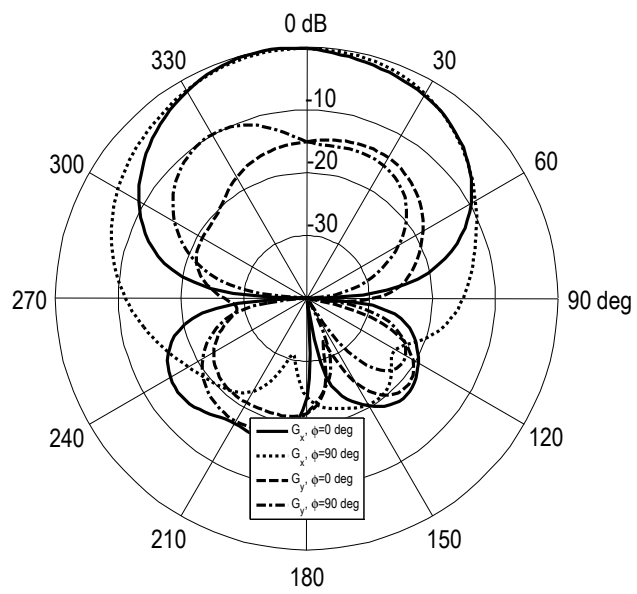

Fig. 7. Single-band Tx antenna, simulated co-polar and cross-polar radiation patterns in the two main plane cuts.

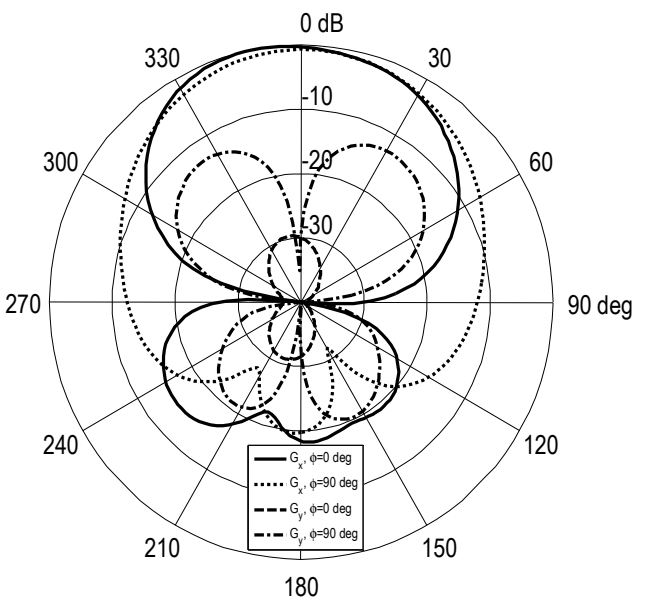

Fig. 8 Dual-band antenna in Rx mode, simulated co-polar and cross-polar radiation patterns in the two main plane cuts. 
plane and coupled to a microstrip line printed in the lower part of the stack-up. Each antenna is thus linearly polarized being the Tx and Rx polarization orthogonal to each other. Dual polarization or circular polarization, thought already demonstrated in [18], [19] for similar antenna configurations, was not taken into account for this work. Interestingly, the dual-band cell is fed through two independent microstrip lines thus resulting in a self-duplexing behavior which is highly effective in the design of the array beam forming network [15]. The material chosen for the design is a Rogers Multiclad HF with $\varepsilon_{e}=3.45$ and $\tan \delta=0.009$. The overall thickness is $1.5 \mathrm{~mm}$. A dielectric cover of Fastrise DS $0.250 \mathrm{~mm}$ thick is placed on the radiating surface. Notice that some of the vias are indented into the slot to reduce the cell size. The configurations shown in Fig. 4 and 5 were analyzed as isolated cells using a commercial finite element simulator [20]. The size of the single-band and dual-band cells are taken with $a_{T X}=a_{R X}=$ $7.5 \mathrm{~mm}$. The simulated [20] radiation patterns for the two antenna types are shown in Fig. 7-9. The single-band Tx antenna presents (Fig. 7) a broad beam with a peak gain of $4.83 \mathrm{dBi}$ and cross-polarization isolation greater than $30 \mathrm{~dB}$ in the broadside direction. It is important to note that the $\mathrm{Tx}$ radiation properties remain almost unchanged from the single-band to the dual-band design though the latter configuration presents a slight degradation of the polarization properties. The Rx radiator in the dual-band cell has a gain of $4.6 \mathrm{dBi}$ and cross-polar radiation greater than $30 \mathrm{~dB}$ in the whole upper hemisphere. As it can be observed in Fig. 10-11, within the $\mathrm{Rx}$ and $\mathrm{Tx}$ bands the gain variation of both antennas is less than $0.5 \mathrm{dBi}$. In the same figures, are also reported the reflection coefficients and, for the dual-band configuration, the coupling between the Tx and Rx radiators. The geometry of both antennas was optimized to obtain good impedance match to $50 \mathrm{Ohm}$ in the whole bands without requiring additional matching networks. As noticed in the previous section, the two antennas are highly isolated especially in the lower band because at this frequency the inner radiator is in cut-off.

\section{ARRAY DESIGN}

The radiating elements were designed in view of their integration in a dual-band beam steering array implemented through the chips introduced in [13]-[15]. As discussed in [21], the proper implementation of this type of architecture requires adopting a complex multilayer stack-up comprising different types of vertical transitions. In particular, the reduced grid size leaves limited routing space to implement the Distribution Network (DN) and to integrate the multicore chips. For this reason, in this work the $\mathrm{DN}$ was split into two parts: a microstrip at the base of the stack-up and a stripline in the intermediate layer SL. A complete stackup used for the design of the array is shown in Fig. 12. The $\mathrm{n}^{\text {th }}$ ground metal layer is indicated with the symbol Gn, while the $\mathrm{n}^{\text {th }}$ dielectric layer with Dn. Each cell is fed through a stripline which is connected to a vertical transition (Via 3 in Fig. 12) to give access to the microstrip ML and to the active devices (MMIC).

\section{A. Stack-up configuration}

In Fig. 13- 14 the cross sectional view is reported along with an

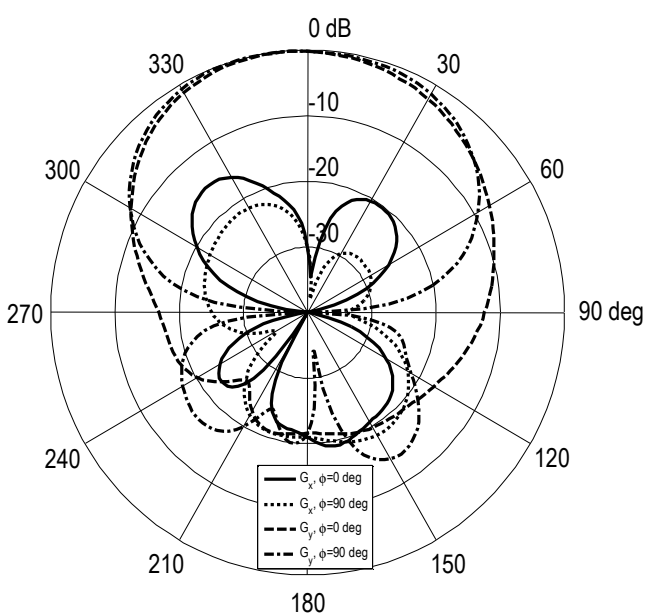

Fig. 9. Dual-band antenna in Tx mode, simulated co-polar and cross-polar radiation patterns in the two main plane cuts.

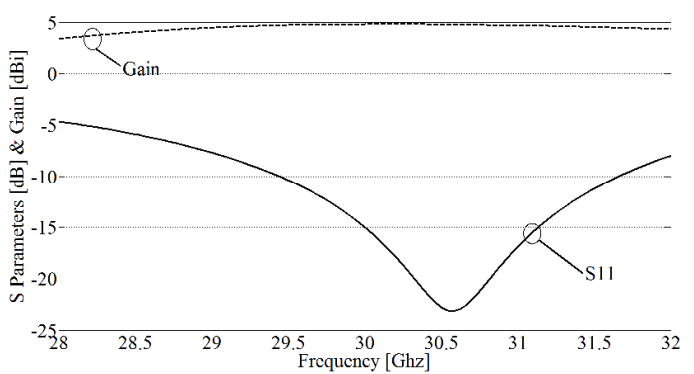

Fig. 10. Simulated single-band Tx antenna: reflection coefficient and gain.

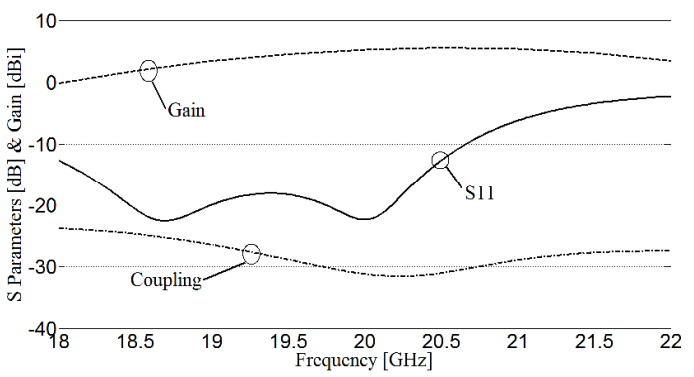

(a)

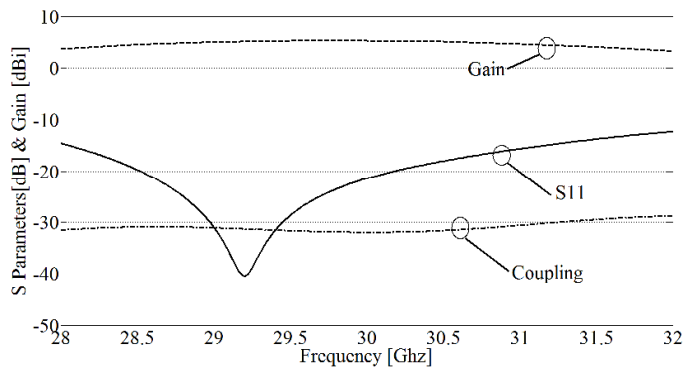

(b)

Fig. 11. Simulated dual-band antenna gain, reflection and transmission coefficient: a) Rx port; b) Tx port.

exploded view of the Dual-Band Cell (DBC) and of the Single-Band Cell (SBC) integrated with a simple DN. 
Dimensions given in the caption are relevant to a radiating element optimized in an infinite array configuration. As it can be noticed, the structure is divided, and fabricated, in two parts: a radiation board (layers D1, G1, D2, G2) and a DN manifold (G3, D3, D4, G4, D5, G5 and D6). The possibility to have two independent stackups allows the implementation of more complex via configurations which will be essential in the design of the final phased array prototype where additional layers have to be included to allocate the digital bus, the control and the bias lines (D5 in Fig. 13). Indeed, by using the conducting adhesive layer the radiation board (RB) and the DN manifold (DNM) can be fabricated with two separate processes making easier the realization of complicated multilayer structure. The two parts are glued together by means of an adhesive conducting foil (Loctite Abelstick $^{\mathrm{TM}}$ ) placed between metal layers G2 and G3. The glued ground planes (G2 and G3) have twin slots through which the radiators are coupled to the feeding striplines. To allow the twin slots to couple, rectangular non-resonant cavities are realized in the conducting adhesive layer as shown in Fig. 12 - 14. A detailed description of the stackup is shown in Fig. 12 while Table II summarizes the proprieties of the three types of via used in the demonstrator presented in this work.

A number of vias have been used between G1 and G2 (see Via 1 in Fig. 12 and Table II) to implement the coaxial structure required for the dual-band and single band radiating elements described in Section III-A. Blind vias between G3 and G4 (see Via 2 in Fig. 12 and Table II) have been used to realize a cage surrounding the striplines and the slots on G3 to avoid the excitation of unwanted parallel plate modes and to reduce coupling between the two slots. The lower part of Figs. 13-14 show the quasi coaxial vertical transition [22] used to connect

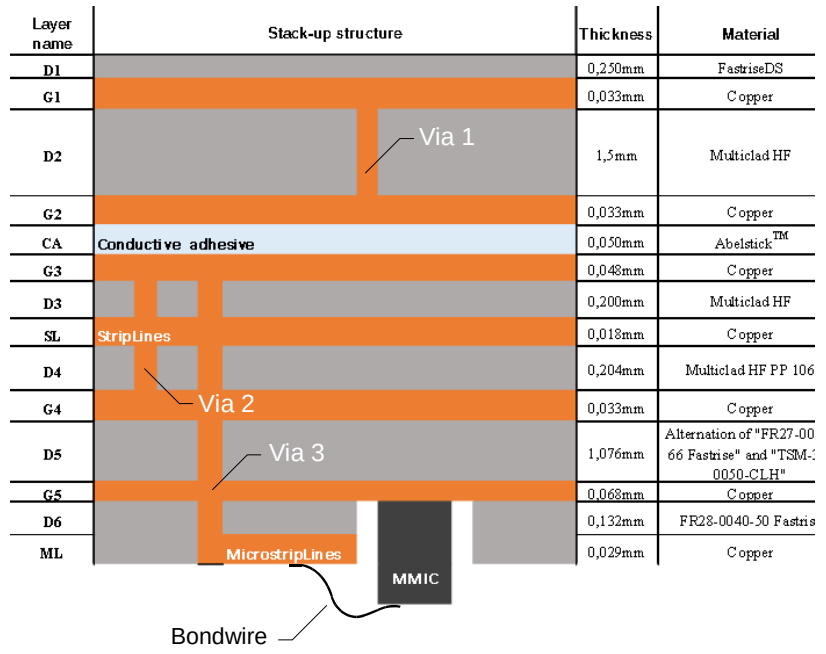

Fig. 12. Description of the array multilayer stackup.

TABLE II

PROPRIETIES OF THE VIA HOLES SHOWN IN FIG. 13

\begin{tabular}{lcc}
\hline \hline Name & Via hole type & $\begin{array}{c}\text { Diameter } \\
{[\mathrm{mm}]}\end{array}$ \\
\hline Via 1 & Through via (RB) & 0.3 \\
Via 2 & Blind via (DNM) & 0.5 \\
Via 3 & Through via (DNM) & 0.25 \\
\hline
\end{tabular}

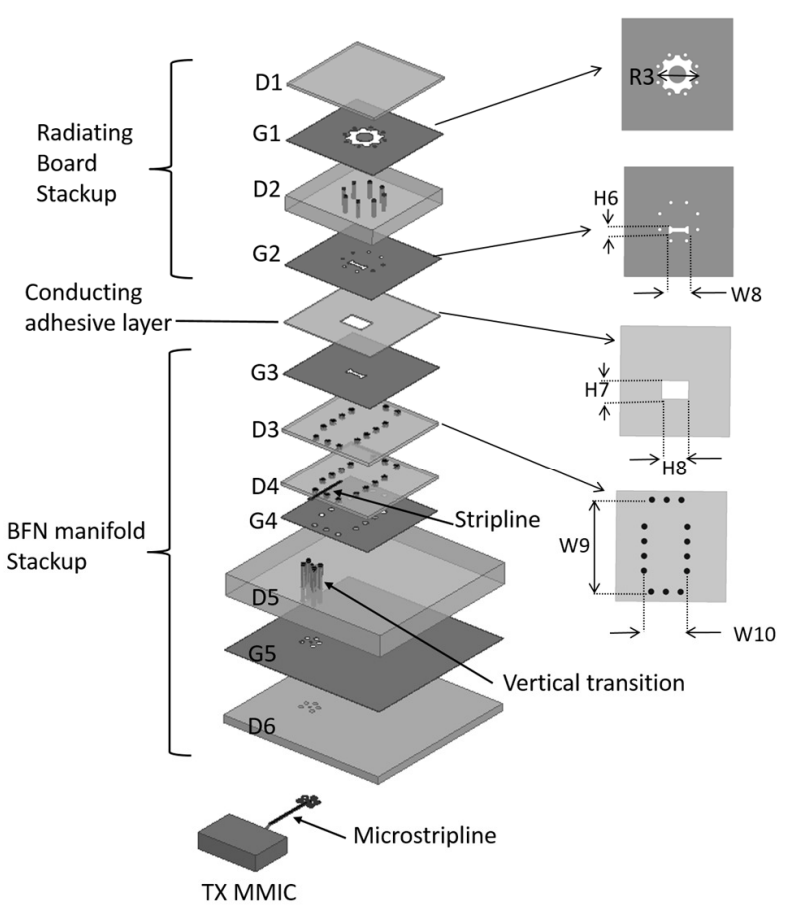

Fig. 13. Exploded 3D view of the Single-Band Cell (SBC) integrated with a simple beam-forming network: $\mathrm{R} 3=3.2 \mathrm{~mm} ; \mathrm{H} 6=0.85 \mathrm{~mm} ; \mathrm{H} 7=1.5 \mathrm{~mm}$; $\mathrm{H} 8=2.1 \mathrm{~mm} ; \mathrm{W} 8=1.6 \mathrm{~mm} ; \mathrm{W} 9=3.5 \mathrm{~mm} ; \mathrm{W} 10=3.1 \mathrm{~mm}$.

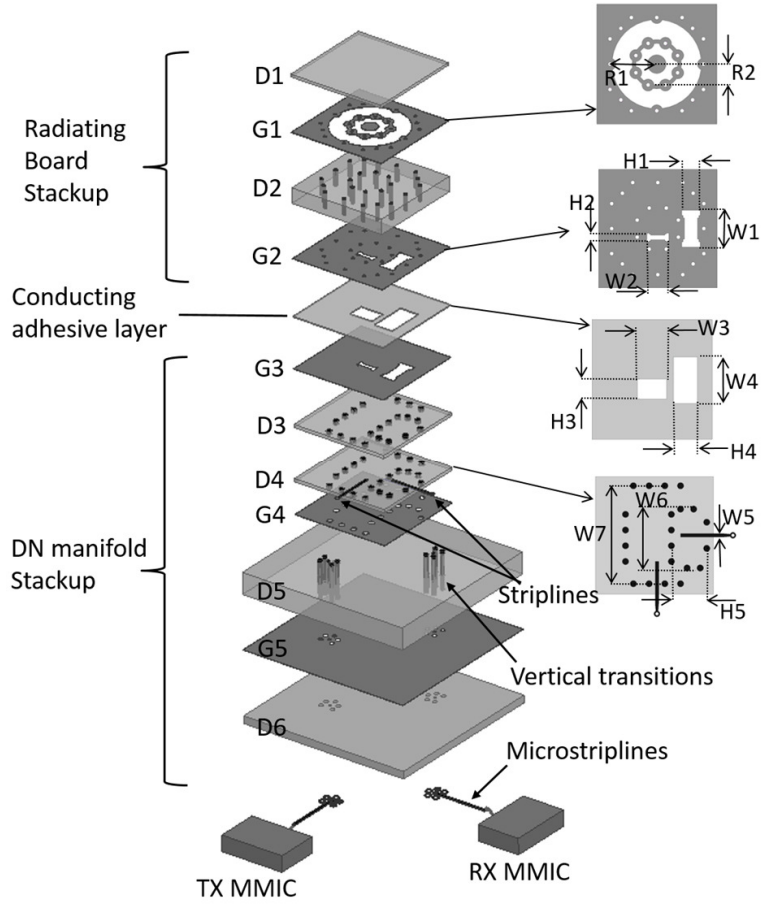

Fig. 14. Exploded 3D view of the dual-band array cells (DBC) integrated with a simple beam-forming network: R1 $=3.5 \mathrm{~mm} ; \mathrm{R} 2=1.7 \mathrm{~mm}$; $\mathrm{H} 1=1.4 \mathrm{~mm} ; \mathrm{H} 2=0.65 \mathrm{~mm} ; \mathrm{H} 3=1.5 \mathrm{~mm} ; \mathrm{H} 4=1.8 \mathrm{~mm} ; \mathrm{H} 5=2.7 \mathrm{~mm} ; \mathrm{W} 1=$ $2.8 \mathrm{~mm} ; \quad \mathrm{W} 2=1.68 \mathrm{~mm} ; \quad \mathrm{W} 3=2.2 \mathrm{~mm} ; \quad \mathrm{W} 4=3.5 \mathrm{~mm} ; \quad \mathrm{W} 5=0.27 \mathrm{~mm}$; $\mathrm{W} 6=4.5 \mathrm{~mm} ; \mathrm{W} 7=7.5 \mathrm{~mm}$ 


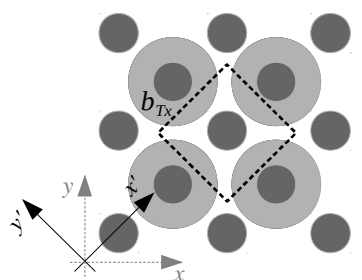

(a)

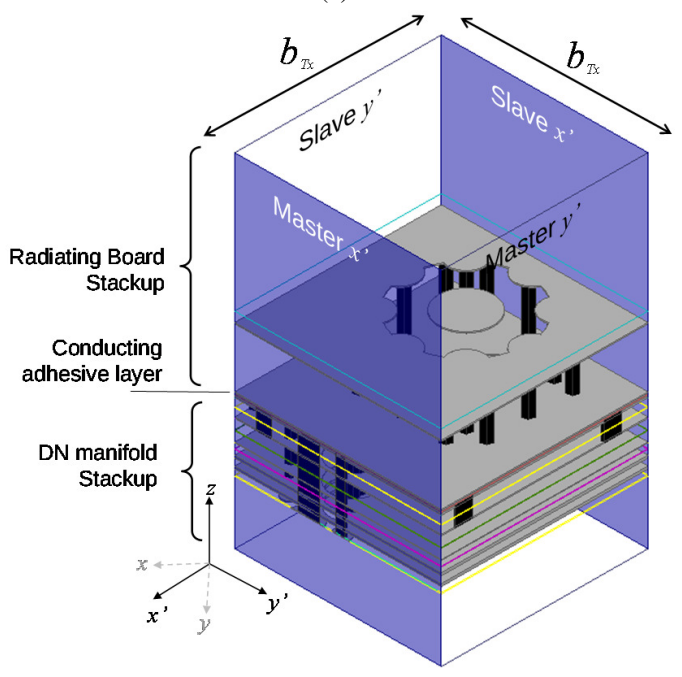

(b)

Fig. 15. Simplified infinite array set-up configurations for the analysis of the Tx radiator. The unit cell is identified rotating the axes around the $z$ axis of 45 deg: a) unit cell definition; b) master/slave condition.

the feeding striplines to the microstrips under D6 where active devices can be placed. Vias between G3 and the layer containing microstrips (Via 3 in Fig. 12 and Table II) have been used to realize the quasi-coaxial transition. In particular, the realization of these transitions requires two different types of vias: grounding vias (from ML to G3) and a central signal via (from ML to SL). In order to reduce the manufacturing complexity, a single type of via (Via3 from ML to G3) has been used for all vias. In a second manufacturing phase, a backdrilling process has been used to remove the unused section (from G3 to SL) of the central signal via.

\section{B. Active impedance analysis}

The active impedance of the proposed radiating elements shown in Figs. 13-14 was evaluated within the entire scanning range by means of HFSS simulations in infinite array configurations. It is worth noticing that, as shown in Fig. 1, the smallest unit cell of the dual-band lattice is constituted by a subarray of three radiators, namely one DBCs and two SBCs. However, the evaluation of the active impedance at various scan angles with this type of cell is not straightforward [23]. In fact, the Floquet analysis applied to this elementary cell would give results valid for the subarray but not for the single element. In particular, in a master-slave linked boundary configuration, like the one used in HFSS, the phases of each radiator in subarray cell can not be directly linked to the phase difference between the vertical master and slave boundaries which are in turn used to identify the scan angle. For this reason, two distinct set-ups, shown in Fig. 15 and 16, were used to validate the array performance.

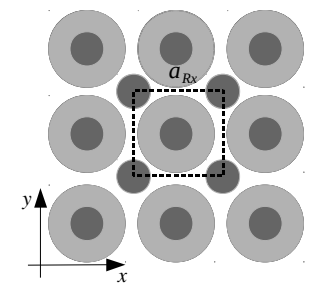

(a)

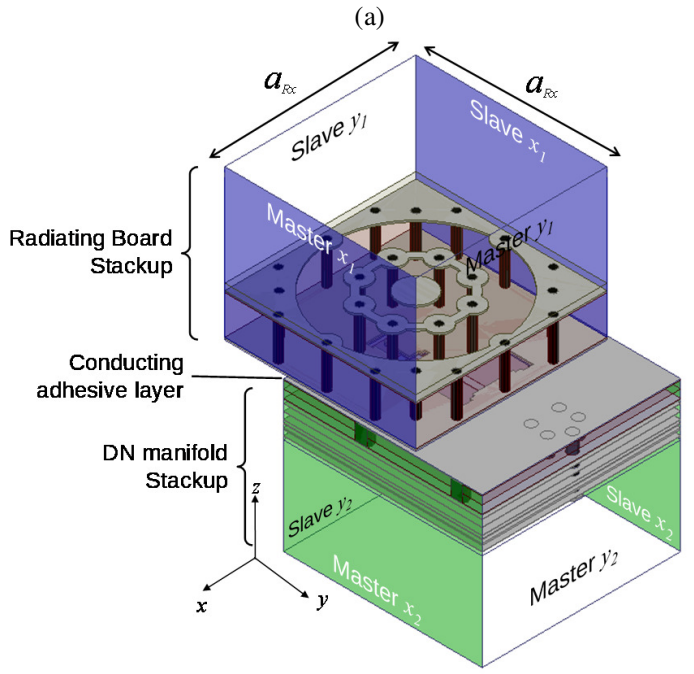

(b)

Fig. 16. Simplified infinite array set-up configuration for the analysis of the $\mathrm{Rx}$ radiator. Two sets of master/slave conditions have been used as the BFN stackup is shifted along the $y$ axis with respect to the radiating boarda) unit cell definition; b) master/slave conditions.

Considering the lattice shown in Fig.1, the Rx radiator was simulated in a DBC configuration with a cell size of $a_{R X}=$ $0.5 \lambda_{20}=7.5 \mathrm{~mm}$ while the Tx radiator was analyzed in a cell having a side of $b_{T X}=5.3 \mathrm{~mm}$ and rotated of $45^{\circ}$ with respect to the main axis. As it will be shown in the next section, although this simulation set-up does not fully reflect the array lattice shown in Fig. 1, it proved to be sufficiently accurate in predicting the input impedance and the radiation characteristics of all radiating elements.

Simulation results in two configurations are shown in Fig. 17 (a)-(d) where the input impedances are represented in the Smith chart for scanning angles $\theta=0^{\circ}, 20^{\circ}, 40^{\circ}, 50^{\circ}, 60^{\circ}$ in the two main planes. As it can be observed, for the Rx element configuration results are acceptable in both $E$ and $H$ planes in the whole scanning range. The Tx element has an excellent matching on the $E$ plane but presents severe mismatch on the $H$ plane at scan angles larger than $30 \mathrm{deg}$. To improve the Tx cell performance, a wide-angle impedance matching layer (WAIM) was introduced as suggested in [24] by adding a dielectric layer of thickness $0.13 \mathrm{~mm}$ and with $\varepsilon_{r}=6.15$, (i.e. Rogers RO3006) $0.94 \mathrm{~mm}$ above the radiating cells. As it can be observed in Fig. 17 (e)-(h), the presence of the WAIM improves the scanning capabilities of the array, in particular on the $\mathrm{H}$ plane, for scan angles down to $60^{\circ}$.

To better appreciate the behavior of the array elements, in figure 18 (a)-(b) and figure 19 (a)-(b) are shown the active reflection coefficients at the input port for the cases presented in Fig. 17 for the case with WAIM layer in place. Spikes due to unwanted resonances [18] are present in the Rx band for scanning angle 


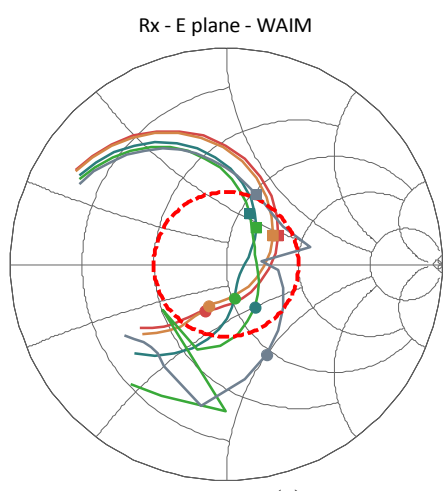

(a)

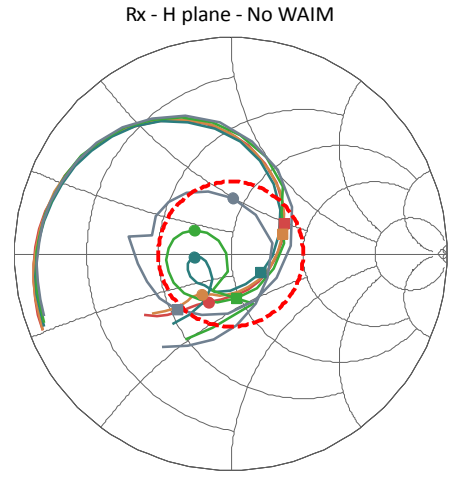

(b)

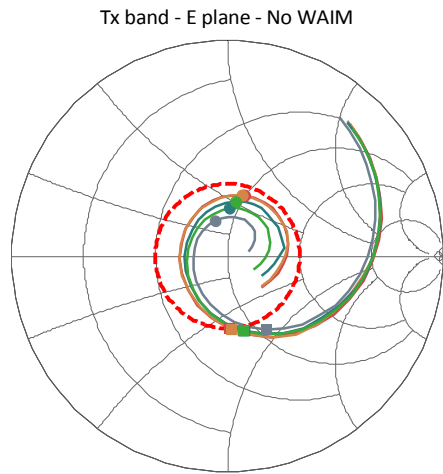

(c)

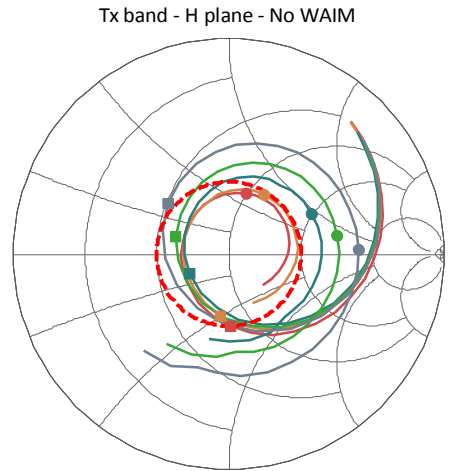

(d)

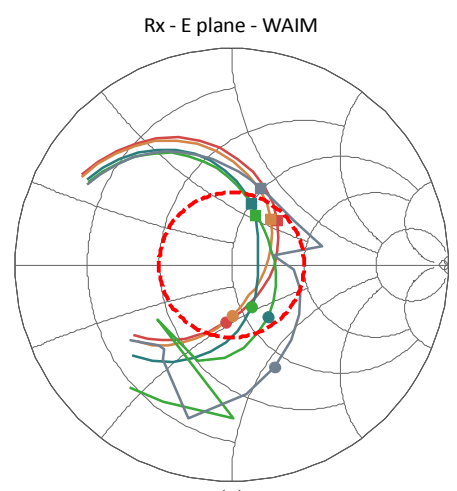

(e)

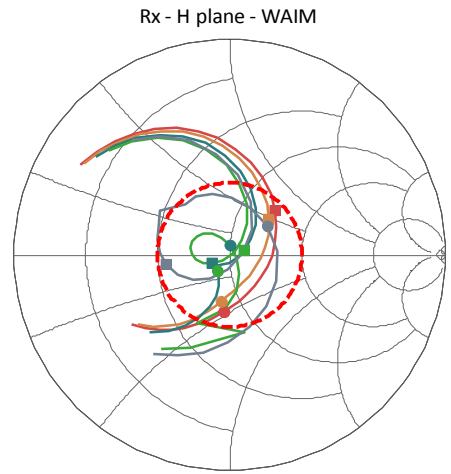

(f)

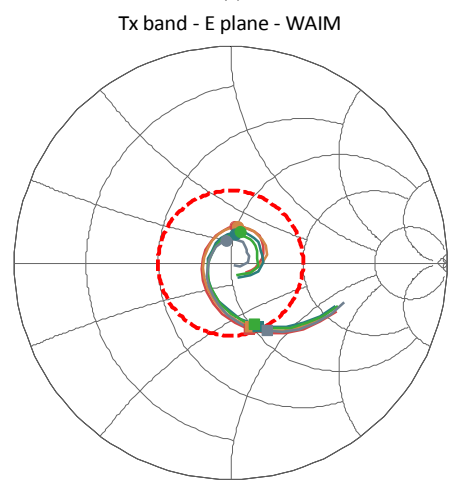

(g)

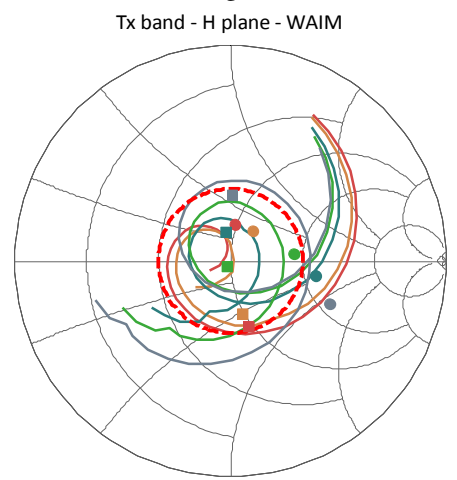

(h)

Fig. 17. Smith Chart representation of the simulated active input impedance of the Rx and Tx elements embedded into an infinite array for different scan angles $\left(\theta_{s}=0^{\circ}, 20^{\circ}, 40^{\circ}, 50^{\circ}, 60^{\circ}\right)$ on the E- and H-planes. Plots in a, b, c, and $\mathrm{d}$ in the left column are obtained without WAIM; plots e, $\mathrm{f}, \mathrm{g}$ and $\mathrm{h}$ in the right column are obtained including a $0.13 \mathrm{~mm}$ thick WAIM layer placed at a distance of $0.94 \mathrm{~mm}$ from D1 and with $\varepsilon_{r}=6.15$ (Rogers RO3006). Square and circular markers indicate start and stop frequencies edges respectively. 


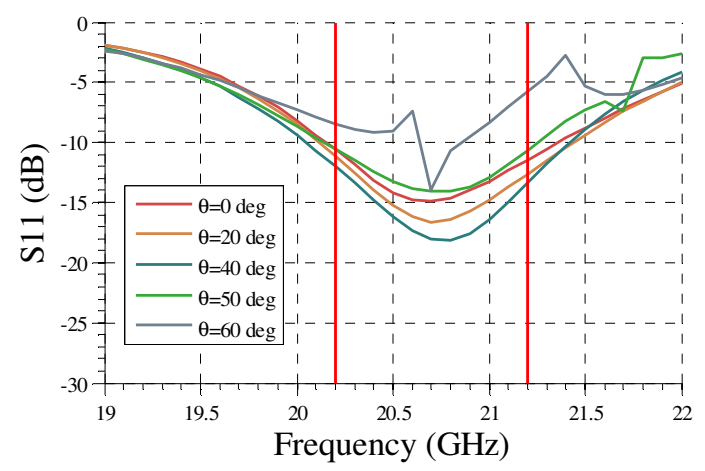

(a)

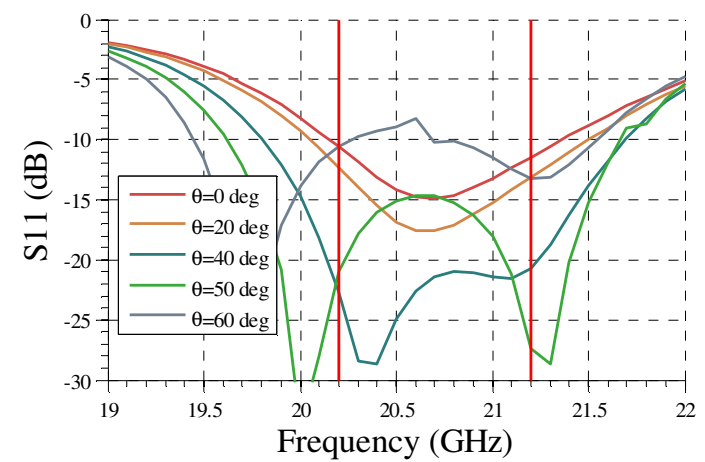

(b)

Fig. 18 Active S11 of the Rx elements scanning on the E plane (a) and on the $\mathrm{H}$ plane (b).

$+-60^{\circ}$ in both $E$ and $H$ planes while, in the Tx band, the antenna is unmatched when scanned to $60^{\circ}$ even if no scan blindness spikes are observed. No further optimization has been attempted to improve scan properties at $60^{\circ}$.

\section{EXPERIMENTAL VALIDATION}

The experimental validation of the scanning performance of the $\mathrm{Rx}$ and Tx antenna elements was carried out measuring their active radiation patterns and evaluating the scanning range as done in [11], [12]. The array prototype used to measure the active patterns is shown in Fig. 20, where the radiators (layer G1 in Fig. 14) are revealed without the dielectric cover. The prototype is composed by a $7 \mathrm{x} 7$ square array of $\mathrm{Rx} / \mathrm{Tx}$ elements interleaved with an array of Tx elements. In total, the array is populated by $49 \mathrm{Rx}-\mathrm{Tx}$ and 56 Tx cells. Only the central dualband array element is connectorized while the other cells are terminated to $50 \mathrm{Ohm}$ chip resistors as shown in Fig. 20-c. This configuration allows to validate the presence of blind angles through the measurement of the active element radiation pattern of the central element embedded into a relevant array environment. In this test scenario, scan blindness in a phased array configuration can be revealed by the presence of nulls measured radiation patterns of the embedded element.

The prototype was built through a sequential build-up PCB process requiring three production stages. In the first and second phase, the radiating board (from D1 to G2) and the BFN manifold (from G3 to D6) were fabricated independently. In a third phase, the two boards were aligned and bonded through a

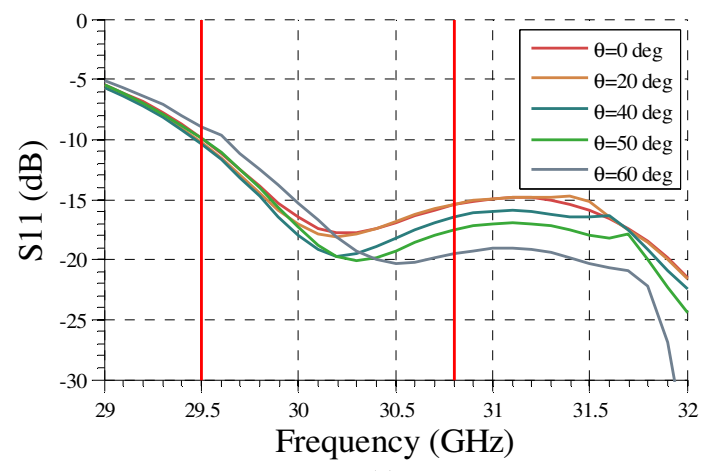

(a)

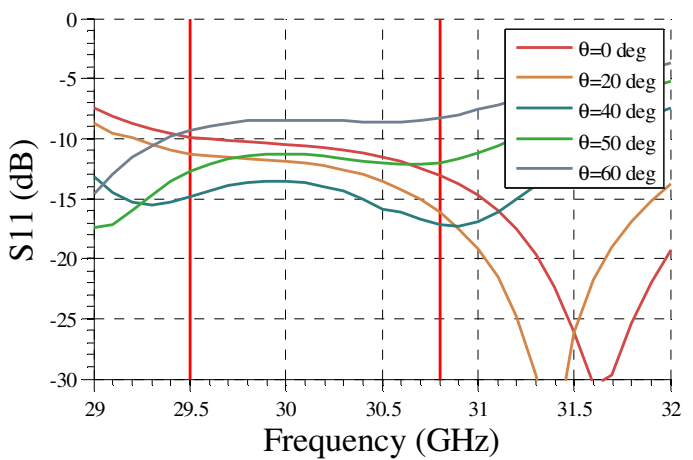

(b)

Fig. 19 Active S11 of the Tx elements scanning on the $E$ plane (a) and on he $H$ plane (b).

conductive adhesive layer, CA, in a thermally controlled press. The layer CA was implemented using the Loctite Abelstik ${ }^{\mathrm{TM}}$ material which provides high electrical and thermal conductivity as well as low curing temperature.

The bonding procedure, involved three types of slots, namely one of the Tx and two of the dual-band antennas. The same slot layout was replicated identically in layers G2 and G3 (Fig. 20b) while slightly larger apertures where created in the adhesive conducting layer CA. The effectiveness of the proposed integration approach was firstly validated through X-rays and cross-section measurements of the bonded layers. As it can be observed in Fig. 21, the maximum measured misalignment between the two boards in the horizontal plan is equal to $19 \mu \mathrm{m}$. It has been verified through simulations that misalignments up to $50 \mu \mathrm{m}$ have no effect on the antenna performance.

The simulated and measured reflection coefficient of the embedded element are shown in Fig. 22 and 23. The Rx and Tx element's performance are well consistent with the numerical results showing a measured bandwidth equal from 20.15 to $21.86 \mathrm{GHz}$ and from 29.6 to $31.28 \mathrm{GHz}$ for the $\mathrm{Rx}$ and Tx case respectively.

The embedded co-polar and cross-polar radiation patterns along the E- and H-plane were measured for the Rx and Tx elements as shown in Fig. 24 and 25 respectively. In both cases, co-polar radiation patterns present broad beams without any null within the hemispherical coverage which in turn results in wide-angle scanning performance. A gain of $4.4 \mathrm{dBi}$ was determined for both the Tx and Rx embedded elements by using the Friis formula and a standard gain horn. In both operational bands the 
cross polarization levels are well below $20 \mathrm{~dB}$ for the $\mathrm{Tx}$ antenna whereas values higher than the simulated ones can be observed in the $\mathrm{Rx}$ antenna in the $\mathrm{H}$ plane. This behavior is ascribed to an imperfect shielding of the outer conductor which deviates the electric field lines. The evaluation of the scanning performance of the arrays was done using the embedded radiation patterns shown in Fig. 24 and 25 to generate array patterns pointing down to $\theta=60^{\circ}$ in both $E$ and $H$ planes. Results, presented in Fig. 26, were obtained simulating a uniformly excited array. Superimposed to the array pattern are the element pattern and the ideal $\cos \theta$ pattern. As it can be seen, grating lobe free patterns are obtained for scan angle up to $50^{\circ}$. At $60^{\circ}$ grating lobes appear in $\mathrm{Rx}$ because cell size is exactly $\lambda / 2$ at $20 \mathrm{GHz}$.

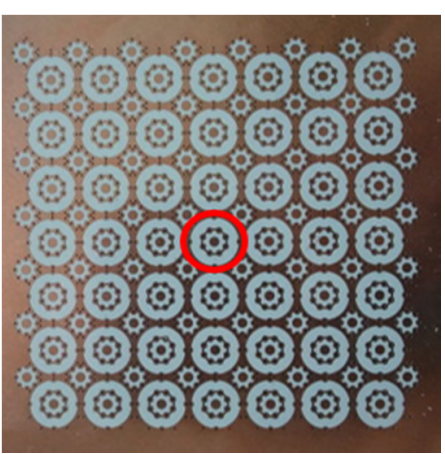

(a)

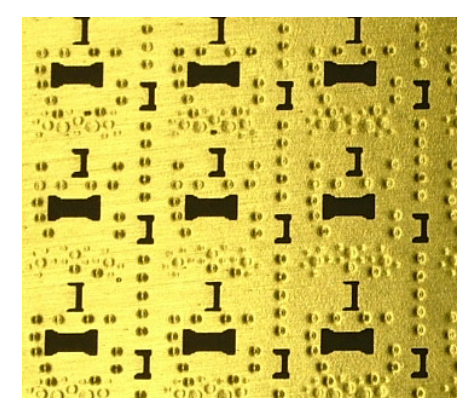

(b)

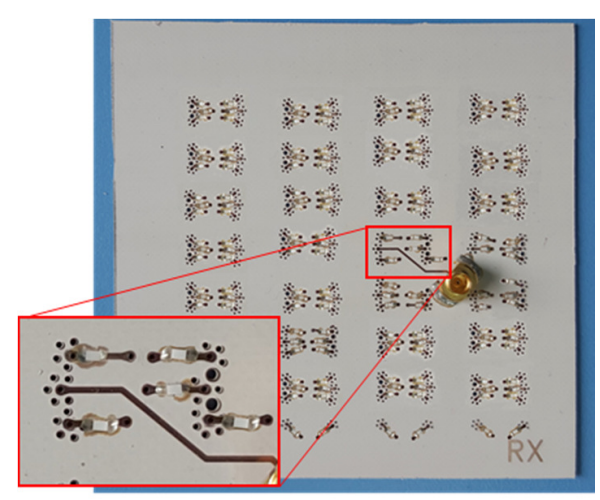

(c)

Fig. 20. Array prototype test structure: a) layer G1; b) layer G2 or G3, blind vias used to create cavities around the slots are also visible; c) bottom view.

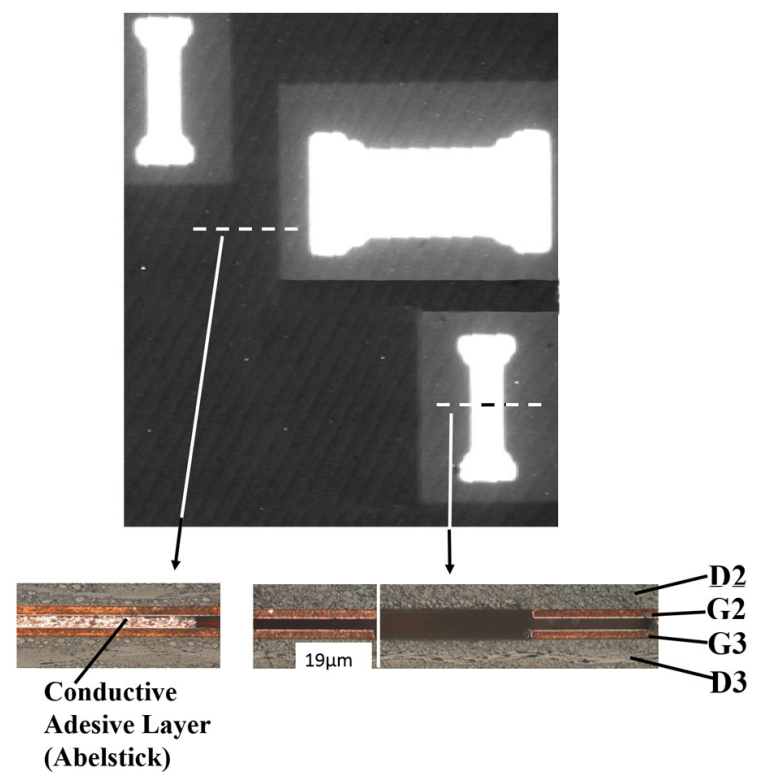

Fig. 21. X-ray image of the conductive adhesive layer between G2 and G3 with two side views of the same layer.

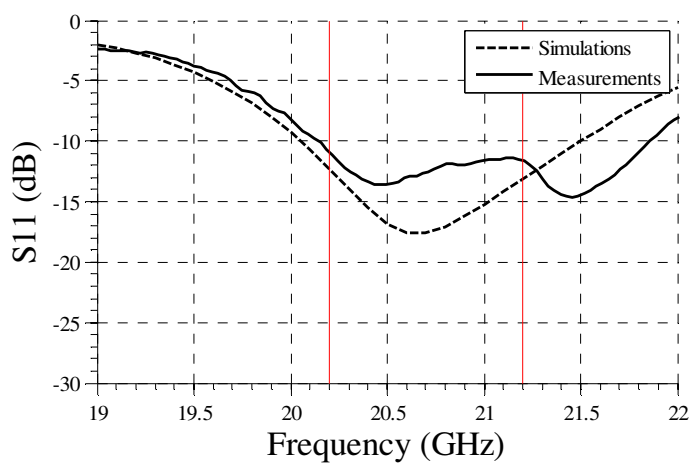

Fig. 22 Return loss of the central Rx element (circled in red) of the array shown in Fig. 20.

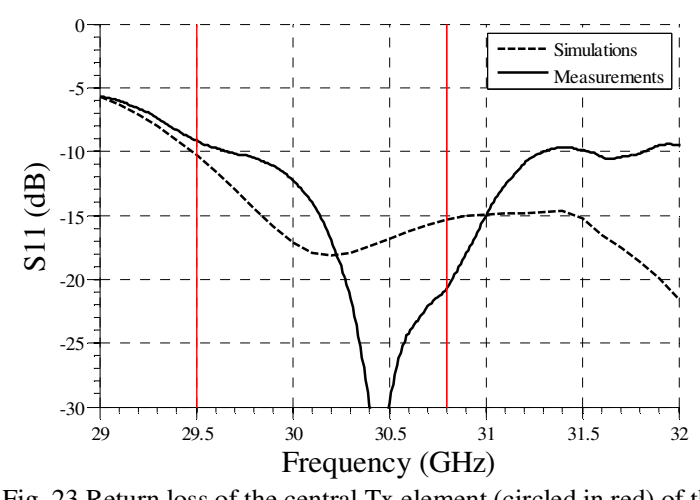

Fig. 23 Return loss of the central Tx element (circled in red) of the array shown in Fig. 20.

\section{CONCLUSIONS}

In this paper a dual band phased array configuration, operating at $\mathrm{K} / \mathrm{Ka}$ band has been studied and experimentally validated. The proposed solution consists of dual-band radiating elements 


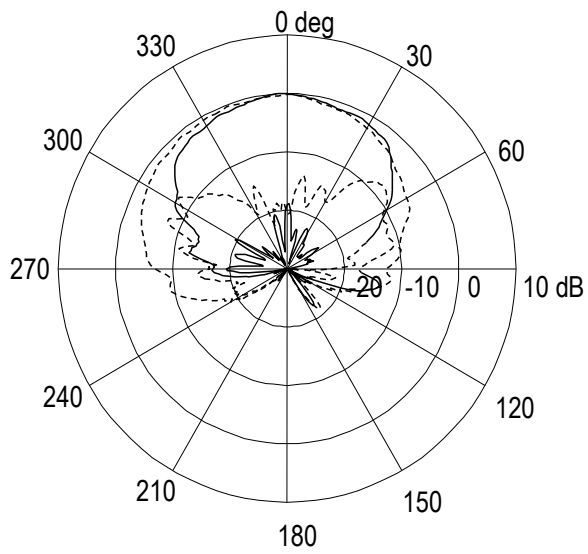

Fig. 24 Co-polar and cross-polar radiation patterns at $20 \mathrm{GHz}$ of the central Rx element of the array shown in Fig. 21-a. Solid line: E-plane; dashed line: H-plane.

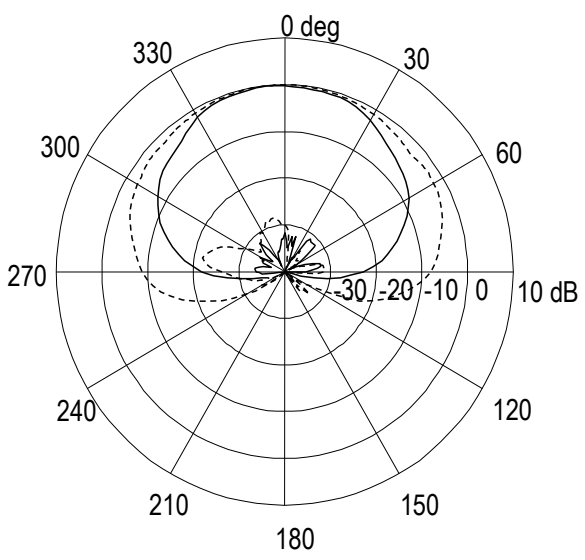

Fig. 25 Co-polar and cross-polar radiation patterns at $30 \mathrm{GHz}$ of the central Tx element of the array shown in Fig. 21-a. Solid line: E-plane; dashed line: H-plane.

interleaved with single band elements. The interleaved configuration limits the number of elements in the array and it avoids the use of multiband or wideband radiators which may be cumbersome to design and to integrate. The radiating elements are designed to be integrated with multicore SiGe BiCMOS chips and they are optimized to scan the beam to more than $50^{\circ}$ in both the $\mathrm{E}$ plane and the $\mathrm{H}$ plane. A new integration technique was introduced to enable more complex and dense PCB configuration. With the proposed integration technique it is possible to split the realization of the radiation board and of the BFN manifold in two separate processes, facilitating the realization of the antenna and the integration of the multicore chips. While the paper focused on $\mathrm{K} / \mathrm{Ka}$ band satellite communications, the solution proposed has a more general validity and it may be applied to different applications which require dual band highly integrated arrays with a large bands separation.

\section{ACKNOWLEDGMENT}

The authors are thankful to Prof. Steven Gao and the staff of the Antenna Laboratory at the University of Kent (UK) for their support in antenna measurements.

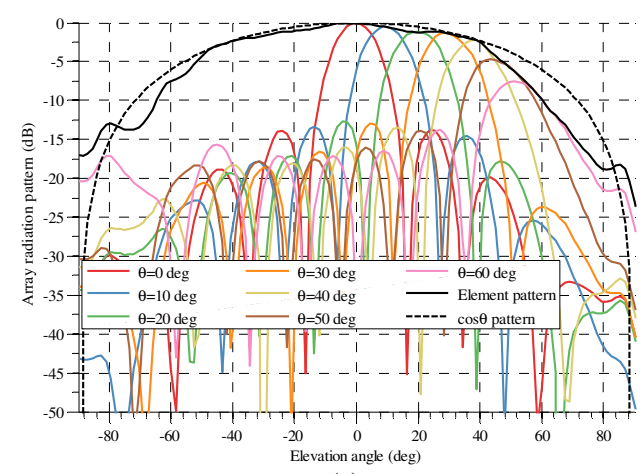

(a)

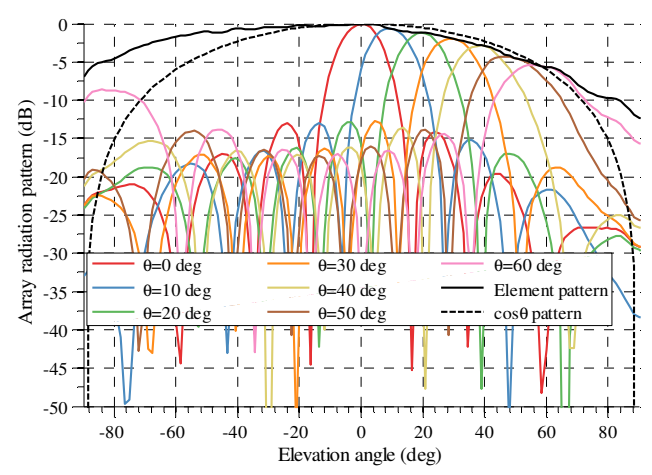

(b)

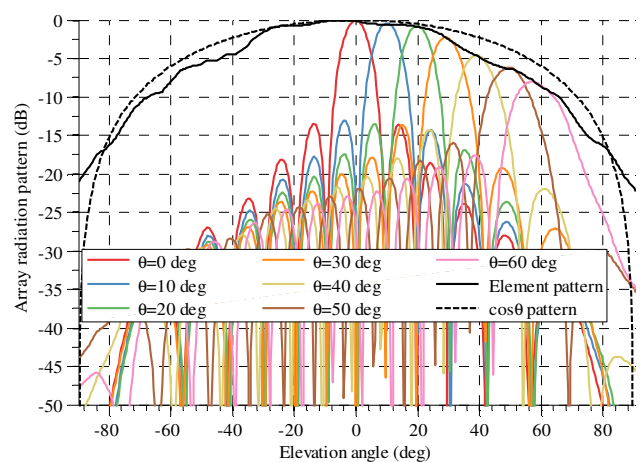

(c)

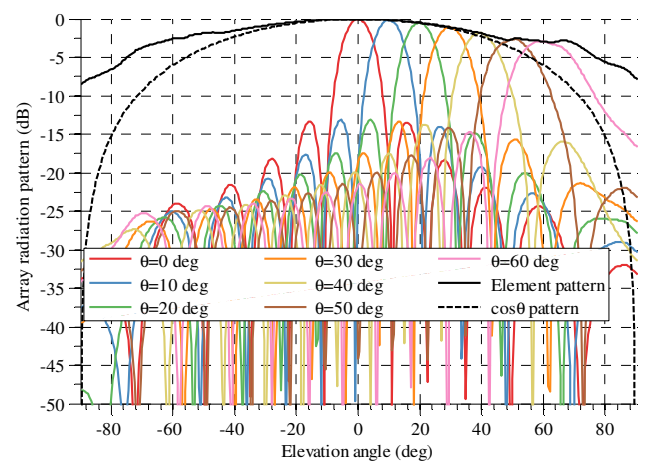

(d)

Fig. 26 Array scanning performance based on the measured embedded element patterns: a) scanning along the E-plane at $20 \mathrm{GHz}$; b) scanning along the $\mathrm{H}$-plane at $20 \mathrm{GHz}$; c) scanning along the E-plane at $30 \mathrm{GHz}$; d) scanning along the H-plane at $30 \mathrm{GHz}$. 


\section{REFERENCES}

[1] W. A. Imbriale, S. Gao, and L. Boccia, Space Antenna Handbook. Wiley-Blackwell, 2012.

[2] W. Hong, A. Goudelev, K. Baek, V. Arkhipenkov, and J. Lee, "24-Element Antenna-in-Package for Stationary 60GHz Communication Scenarios," IEEE Antennas Wirel. Propag. Lett., vol. 10, pp. 738-741, 2011.

[3] D. Liu and Y. P. Zhang, "Integration of Array Antennas in Chip Package for 60-GHz Radios," Proc. IEEE, vol. 100, no. 7, pp. 2364-2371, Jul. 2012.

[4] W. Roh, J.-Y. Seol, J. Park, B. Lee, J. Lee, Y. Kim, J. Cho, K. Cheun, and F. Aryanfar, "Millimeter-wave beamforming as an enabling technology for $5 \mathrm{G}$ cellular communications: theoretical feasibility and prototype results," IEEE Commun. Mag., vol. 52, no. 2, pp. 106 113, Feb. 2014.

[5] T.-H. Chio and D. H. Schaubert, "Parameter study and design of wide-band widescan dual-polarized tapered slot antenna arrays," IEEE Trans. Antennas Propag., vol. 48, no. 6, pp. 879-886, Jun. 2000.

[6] J. A. Kasemodel, C.-C. Chen, and J. L. Volakis, "Wideband Planar Array With Integrated Feed and Matching Network for Wide-Angle Scanning," IEEE Trans. Antennas Propag., vol. 61, no. 9, pp. 4528-4537, Sep. 2013.

[7] D. Cavallo, G. Gerini, R. Bolt, D. Deurloo, R. Grooters, A. Neto, G. Toso, and R. Midthassel, "Ku-band dualpolarized array of connected dipoles for satcom terminals: Theory and hardware validation," in 2013 7th European Conference on Antennas and Propagation (EuCAP), 2013, pp. 459-460.

[8] S. Gao, F. Qin, C. Mao, A. Patyuchenko, M. Younis, G. Krieger, S. Glisic, W. Debski, L. Boccia, G. Amendola, E. Arnieri, M. Krstic, A. Koczor, P. Penkala, and E. Celton, "A Ka/X-band digital beamforming synthetic aperture radar for earth observation," in $20157 \mathrm{th}$ International Conference on Recent Advances in Space Technologies (RAST), 2015, pp. 681-686.

[9] R. Pokuls, J. Uher, and D. M. Pozar, "Microstrip antennas for SAR applications," IEEE Trans. Antennas Propag., vol. 46, no. 9, pp. 1289-1296, Sep. 1998.

[10] H. Zhai, Q. Gao, C. Liang, R. Yu, and S. Liu, "A DualBand High-Gain Base-Station Antenna for WLAN and WiMAX Applications," IEEE Antennas Wirel. Propag. Lett., vol. 13, pp. 876-879, 2014.

[11] S. E. Valavan, D. Tran, A. G. Yarovoy, and A. G. Roederer, "Dual-Band Wide-Angle Scanning Planar Phased Array in X/Ku-Bands," IEEE Trans. Antennas Propag., vol. 62, no. 5, pp. 2514-2521, May 2014.

[12] S. E. Valavan, D. Tran, A. G. Yarovoy, and A. G. Roederer, "Planar Dual-Band Wide-Scan Phased Array in X-Band," IEEE Trans. Antennas Propag., vol. 62, no. 10, pp. 5370-5375, Oct. 2014.

[13] L. Boccia, A. Shamsafar, E. Arnieri, A. I. Sandhu, G. Amendola, T. Purtova, F. Tarabani, V. Valenta, H. Schumacher, E. Meniconi, M. Kaynak, B. Tillack, S. Gao, and V. Ziegler, "SiGe BiCMOS Technology for KaBand Satcom on the Move User Terminals," in The 8th European Conference on Antennas and Propagation
(EuCAP 2014) (EuCAP 2014), The Hague, The Netherlands, 2014.

[14] L. Boccia, A. Shamsafar, E. Arnieri, A. Sandhu, G. Amendola, T. Purtova, X. Gai, V. Valenta, H. Schumacher, W. Menzel, E. Meniconi, M. Kaynak, B. Tillack, and V. Ziegler, "SatCom Rx/Tx User Terminal Antennas with Intelligent Pixels," ESA Workshop SatCom User Termin., 2012.

[15] Y. T. Lo and S. W. Lee, Eds., Antenna Handbook. Boston, MA: Springer US, 1988.

[16] G. Amendola, E. Arnieri, L. Boccia, and V. Ziegler, "Annular ring slot radiating element for integrated millimeter wave arrays," in Proceedings of 6th European Conference on Antennas and Propagation, EuCAP 2012, 2012, pp. 3082-3085.

[17] A. K. Bhattacharyya, A. R. Cherrette, and R. D. Bruno, "Analysis of Ring-Slot Array Antenna Using Hybrid Matrix Formulation," IEEE Trans. Antennas Propag., vol. 61, no. 4, pp. 1642-1650, Apr. 2013.

[18] F. Greco, G. Amendola, E. Arnieri, and L. Boccia, "A Dual-Band, Dual-Polarized Array Element for Ka Band Satcom on the Move Terminals," in The 8th European Conference on Antennas and Propagation (EuCAP 2014) (EuCAP 2014), The Hague, The Netherlands, 2014.

[19] A. Shamsafar, L. Boccia, T. Purtova, F. Tabarani, E. Arnieri, G. Amendola, and H. Schumacher, "A Four-Port SiGe BiCMOS Duplexer for Ka-Band SatCom on the Move User Terminals," IEEE Microw. Wirel. Compon. Lett., vol. PP, no. 99, pp. 1-1, 2015.

[20] Ansys HFSS, v. 2015. .

[21] E. Meniconi, V. Ziegler, R. Sorrentino, and T. Chaloun, "3D integration technologies for a planar dual band active array in Ka-band," in Microwave Conference (EuMC), 2013 European, 2013, pp. 215-218.

[22] E. R. Pillai, "Coax via-A technique to reduce crosstalk and enhance impedance match at vias in high-frequency multilayer packages verified by FDTD and MoM modeling," IEEE Trans. Microw. Theory Tech., vol. 45, no. 10, pp. 1981-1985, Oct. 1997.

[23] M. H. Awida, A. H. Kamel, and A. E. Fathy, "Analysis and Design of Wide-Scan Angle Wide-Band Phased Arrays of Substrate-Integrated Cavity-Backed Patches," IEEE Trans. Antennas Propag., vol. 61, no. 6, pp. 30343041, Jun. 2013.

[24] E. Magill and H. A. Wheeler, "Wide-angle impedance matching of a planar array antenna by a dielectric sheet," in Antennas and Propagation Society International Symposium, 1965, 1965, vol. 3, pp. 164-169. 


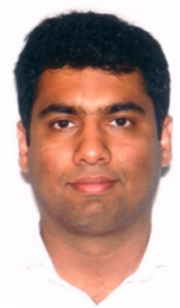

Ali Imran Shandu received the B.S. degree in electronics engineering from COMSATS Institute of Information Technology (CIIT), Pakistan, in 2007 and the M.S. degree in communication engineering from CHALMERS University of Science and Technology, Gothenburg, Sweden in 2010. He is currently pursuing the Ph.D. degree in electrical engineering at the Division of Computer, Electrical, and Mathematical Sciences and Engineering (CEMSE), King Abdullah University of Science and Technology (KAUST), Thuwal, Saudi Arabia.

Since September 2007, he has been a Lecturer with the Department of Electrical Engineering, CIIT Lahore, Pakistan. From September 2011 to March 2013, he worked as a Research Engineer with the Microwave Lab, University of Calabria, Italy. His research interests include inverse electromagnetic scattering problems with emphasis on sparsity-promoting regularization schemes and micro-strip antennas.

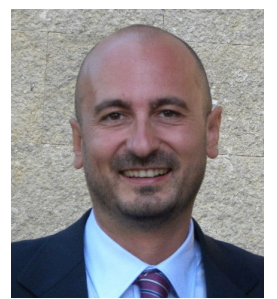

Emilio Arnieri was born in Cosenza, Italy, in 1977. He received the degree (with honors) in Information Technology Engineering from the University of Calabria, Rende, Italy, in 2003 and the Ph.D. Degree in Electronic Engineering from the University "Mediterranea" of Reggio Calabria, in 2007. Currently, he is an Assistant Professor with the Department of Informatics, Modeling, Electronics and System Engineering (DIMES), University of Calabria (Italy). His main research activities concern the development of dual-band antennas and millimeter-wave components, synthetic aperture radar (SAR), development of numerical methods for the electromagnetic modeling of microwave, and millimeter-wave circuits (substrate integrated circuits, slotted substrate-integrated waveguide arrays, and substrate-integrated waveguide resonators).

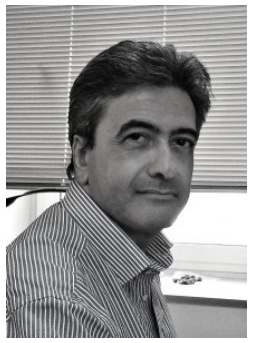

Giandomenico Amendola (M'96, SM '15) received the Electrical Engineering degree from the Università della Calabria, Rende (CS), Italy, in 1987. From 1988 to 1992 , he was a Research Fellow with the Proton Synchrotron Division, European Center for Nuclear Research (CERN), Geneva, Switzerland. He is with the Dipartimento di Elettronica, Informatica e Sistemistica, Università della Calabria, where he is currently an Associate Professor. His main research interests are in the area of antennas, phased arrays and microwave and millimeter-wave circuits.

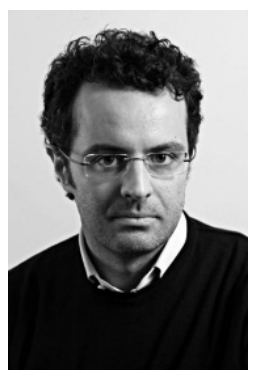

Luigi Boccia (S'00 - M'03) was heart born in Lungro, Italy, in 1975. He received the degree in Information Technology Engineering from the University of Calabria, Rende, Italy, and the Ph.D in Electronic Engineering from the University "Mediterranea" of Reggio Calabria, Italy, in 2000 and 2003 respectively. Since January 2005 he has been Assistant Professor in electromagnetics at the Faculty of Engineering of the University of Calabria. His current research interests include low-multipath GNSS antennas, reflectarrays, beam scanning antennas, micro- and millimetre-wave IC design. Dr. Boccia is also member of the European Microwave Association (EuMA) and of the Società Italiana di Elettromagnetismo (SIEm). He serves as a technical reviewer for many international journals and conferences. He is the co-editor of the "Space Antenna Handbook" (Wiley, 2012).

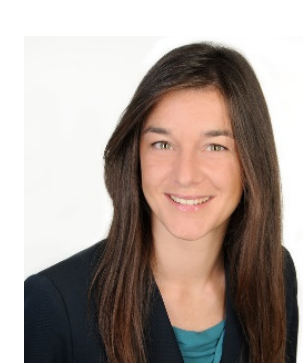

Erika Meniconi was born in Perugia, Italy on July 13, 1983. In 2007 she received her bachelor degree in Information Engineering, in 2010 her M.Sc. degree (with distinction) in Electronic Engineering and in 2015 her $\mathrm{PhD}$ title from the University of Studies of Perugia, Italy. For her bachelor thesis research, she worked on phase shifters for reconfigurable antennas at the Department of Electronic and Information Engineering (DIEI) of the University of Perugia, Italy. Her Master thesis was focused on RFMEMS based switching modules for Ka-band applications and was carried out at the research department of Airbus Defence and Space GmbH, Ottobrunn, Germany.

From 2010 till 2014, she successfully worked on her Ph.D. at Airbus Defence and Space GmbH, Ottobrunn, Germany, in cooperation with the University of Studies of Perugia (DIEI Department). Her main research activity included design, integration, and characterization of RF-MEMS based subsystems and highly integrated RF-frontends for electronically steerable antennas. Since end of 2014 she is working as Senior Design Engineer at TriQuint Semiconductor $\mathrm{GmbH}$, a company of Qorvo, in Munich, Germany.

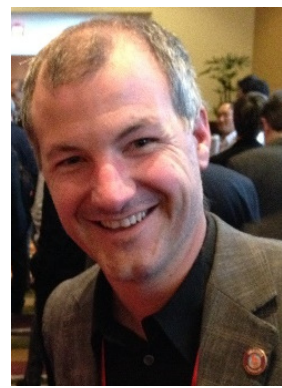

Volker Ziegler received his Dipl.-Ing. degree in electrical engineering and his Dr.-Ing. degree (with honors) both from the University of Ulm, Germany, in 1997 and 2001, respectively. From 2002 to 2003, he was member of the "Knowledge Exchange Group for Research and Technology" at the DaimlerChrysler AG in Stuttgart, Germany. During this trainee period, he was working at the University of Michigan, Ann Arbor, USA on the design of GaN high-power MMICs and at United Monolithic Semiconductors, Orsay, France on the modeling and characterisation of III-V semiconductor devices. Afterwards, he joined EADS Innovation Works, Ottobrunn, Germany, where he became an EADS Expert for "Microwave Technologies and Systems" in 2007. From 2013 to 2015, he was the Head of Team "RF and Waveforms" within AIRBUS Group Innovations. Since 2016, he is the Head of Team "Automatic Flight Systems", responsible for the research performed in the field of automated systems including integrated perception, trajectory optimization, image processing and virtual reality for human-machine-interfaces.

Volker Ziegler is senior member of the IEEE, member of the IEEE MTT-S Technical Coordinating Committee 21 on RF-MEMS and member of the IEEE MTT Antennas \& Propagation German Chapter Executive Board. He served twice as Associated Editor for the „International Journal of Microwave and Wireless Technologies" and was for several years a member of the Technical Program Committee for the European Microwave Week. He authored or coauthored more than 70 papers, holds eleven patents and is an industrial advisor of the "Component Technical Board on Microwaves" for the European Space Agency. 